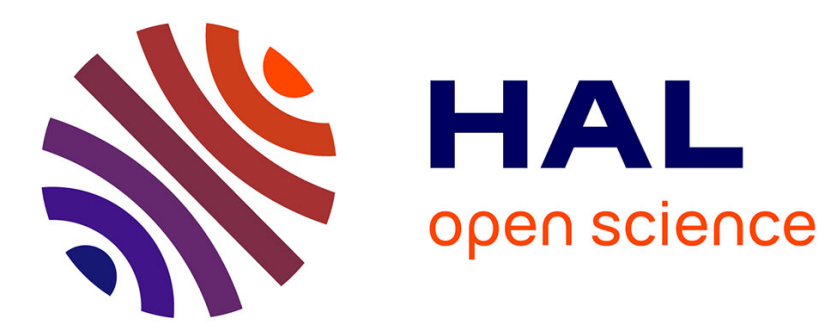

\title{
Expected Utility without Parsimony
}

Antoine Billot, Vassili Vergopoulos

\section{To cite this version:}

Antoine Billot, Vassili Vergopoulos. Expected Utility without Parsimony. 2014. halshs-01021392

\section{HAL Id: halshs-01021392 \\ https://shs.hal.science/halshs-01021392}

Submitted on 9 Jul 2014

HAL is a multi-disciplinary open access archive for the deposit and dissemination of scientific research documents, whether they are published or not. The documents may come from teaching and research institutions in France or abroad, or from public or private research centers.
L'archive ouverte pluridisciplinaire HAL, est destinée au dépôt et à la diffusion de documents scientifiques de niveau recherche, publiés ou non, émanant des établissements d'enseignement et de recherche français ou étrangers, des laboratoires publics ou privés. 


\section{Documents de Travail du Centre d'Economie de la Sorbonne}

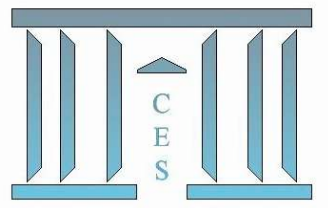

\section{Expected Utility without Parsimony}

Antoine BILlOT, Vassili VERGOPOUlos

2014.48

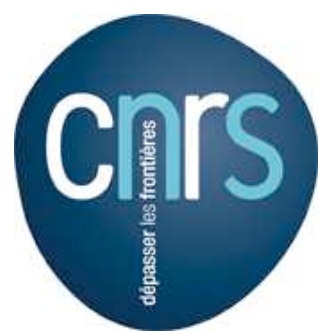

Maison des Sciences Économiques, 106-112 boulevard de L'Hôpital, 75647 Paris Cedex 13 http://centredeconomiesorbonne.univ-paris 1.fr/bandeau-haut/documents-de-travail/ 


\title{
Expected Utility without Parsimony
}

\author{
Antoine Billot* and Vassili Vergopoulos ${ }^{\dagger}$
}

March 19, 2014

\begin{abstract}
This paper seeks to interpret observable behavior and departures from Savage's model of Subjective Expected Utility (SEU) in terms of knowledge and belief. It is shown that observable behavior displays sensitivity to ambiguity if and only if knowledge and belief disagree. In addition, such an epistemic interpretation of ambiguity leads to dynamically consistent extensions of non-SEU preferences.

Keywords: ambiguity, state of the world, knowledge, dynamic consistency

JEL classification: D81, D83, D90
\end{abstract}

\section{Introduction}

What does a Decision-Maker (DM) actually know about his uncertain environment and to what extent does his knowledge affect his observable behavior? Since Savage (1954), standard decision-theoretic practice assumes that uncertainty is described by a state space. Typically, each possible state is nothing but a possible observation. For instance, in a coin flip, states are usually taken to correspond to the two possible observations, heads and tails. Such a conception of states captures the idea that each state fully determines the observation. This amounts to saying that the DM has a perfect knowledge of states: at each possible state, he knows the observation for sure. However, this conception of states is sometimes too restrictive, as it may happen that the DM fails to infer all logical implications of his knowledge - he is not logically omniscient, see Lipman (1999) — or fails to construct a complete representation of uncertainty - he perceives unforeseen contingencies, see Kreps (1992) or Dekel, Lipman and Rustichini (2001). The common feature of these last situations lies in that they require a more general notion of state allowing the DM

${ }^{*}$ LEMMA-Université Panthéon-Assas, and IuF: billot@u-paris2.fr

†PSE-Université Panthéon-Sorbonne: vassili.vergopoulos@univ-paris1.fr

We wish to thank Alain Chateauneuf, Michèle Cohen, Eric Danan, Itzhak Gilboa, Michel Grabisch, Ani Guerdjikova, Jean-Philippe Lefort, David Schmeidler and Jean-Marc Tallon for stimulating discussions and participants of various seminars, workshops and conferences (University Panthéon-Sorbonne-CES, University of Cergy-Pontoise-THema, University of California at Irvine, PSE-OSE 2012, RUD 2013, SAET 2013) for helpful comments on earlier versions of this paper. 
to doubt the precise observation that corresponds to a given state. In this conception, a state no longer determines the resolution of uncertainty in terms of observations but rather encodes the DM's knowledge about this resolution.

In modern physics, the distinction between states and observations is rather common. For instance, the paradoxical Schrödinger cat generates two possible observations, dead or alive, but is described through three states, $D, A$ and $\Psi$ : at state $A$, the physicist knows that the cat is alive; at state $D$, he knows that the cat is dead and, at state $\Psi$, he simply ignores whether the cat is dead or alive and thus knows only that both observations are possible: in short, at state $\Psi$, he believes both observations. As a result, the set of states at which the cat is known to be alive only contains $A$ while the set of states at which the cat is believed to be alive contains $A$ and $\Psi$. This distinction between knowledge and belief only emerges when there are states at which doubts are allowed-which are therefore called ambiguous states. Indeed, if one removes state $\Psi$ from the state space, then knowledge and belief agree. This form of ambiguity is called state ambiguity to distinguish it from the classical ambiguity of Ellsberg (1961).

The following example makes it clear that the disagreement between knowledge and belief has behavioral implications. Consider the two bets $f$ and $g$ defined in the figure below as mappings from observations, dead or alive, onto monetary outcomes, $\$ 0, \$ 1$ or $\$ 100$.

\begin{tabular}{ccc}
\hline & dead & alive \\
\hline$f$ & $\$ 0$ & $\$ 100$ \\
\hline$g$ & $\$ 1$ & $\$ 0$ \\
\hline
\end{tabular}

It is natural to expect the physicist to choose bet $g$ when he knows that the cat is dead and to choose bet $f$ when he believes that the cat is dead since this means that the cat might be alive as well- the observation alive is no longer counterfactual.

The main purpose of this paper is to construct a decision model that is based on a distinction between states and observations and employs knowledge and belief to explain behavior. More precisely, the DM is assumed to conform to subjective Expected utility (SEU) of Savage (1954) with respect to (wrt) the set of states. ${ }^{1}$ In addition, the Savage theory is enriched with two more primitive notions: a set of observations and a function, called an interpretation, that maps acts defined over observations onto acts defined over states. These additional terms first induce observable preferences wrt the set of observations and, second, they capture the DM's knowledge. Therefore, they make it possible to study the observable behavioral implications of knowledge.

A first result shows that the DM's observable preferences are SEU if and only if knowledge and belief do agree - a situation called parsimony -(Proposition 2). Put differently, departures from SEU and, in particular, sensitivity to classical ambiguity are equivalent to the disagreement between knowledge and belief: there are states at which the DM considers that some event is possible but he does not know this event for sure. In short, classical ambiguity emerges if and only if state ambiguity occurs. Second, even in the latter situation, the restriction of the DM's preferences to the subdomain over which knowledge and belief

\footnotetext{
${ }^{1}$ For convenience, it is sometimes said that 'the DM's preferences are SEU'.
} 
agree necessarily conforms to SEU (Theorem 1). Finally, beyond this subdomain, preferences may contradict expected utility maximization and be, for instance, of the choquet Expected Utility (CEU) type axiomatized by Schmeidler (1989) (Theorem 2). These results are applied to the foundational issue of dynamic consistency met by ambiguity. It is wellknown in the literature that the DM behaves consistently in dynamic choice if and only if his preferences satisfy the sure Thing Principle (STP). Savage (1954, Ch. 2, §7) writes: 'The sure-thing principle cannot appropriately be accepted as a postulate (...), because it would introduce new undefined technical terms referring to knowledge and possibility that would render it mathematically useless without still more postulates governing these terms.' It is here suggested that the introduction of knowledge and belief (or possibility) into the decision process would lead to a deeper understanding of STP and, therefore, of dynamic consistency. Actually, knowledge and belief are shown, in a final result, to give rise to a compelling form of dynamic consistency that is always achievable even in case of ambiguity (Theorem 3). Furthermore, the standard form of dynamic consistency is obtained as a particular case when knowledge and belief agree. This finally explains the incompatibility between standard dynamic consistency and ambiguity, since the latter precisely reveals the disagreement between knowledge and belief.

Several papers in the literature study the implications of knowledge on behavior. For instance, the decision model of Mukerji (1997) can be viewed as the case obtained through a particular specification of the interpretation relating acts over observations to acts over states (see Example 2 below). This specification forces behavior to be consistent with CEU wrt to belief functions only, the latter being a particular case of capacities. In contrast, the interpretations considered throughout the following pages are not specified but only required to conform to a set of axioms and, therefore, behavior could be consistent with any capacity, as well as multiple priors à la Gilboa and Schmeidler (1989). Incidentally, this more general approach suggests a reinterpretation of belief functions as beliefs of individuals that are maximally averse to state ambiguity. Moreover, while Dominiak (2008) provides epistemic foundations of ambiguity within the formal model of knowledge known as S5, this paper rather aims at explaining ambiguity within the SEU model and relies upon a more intuitive model of knowledge directly inspired by the preference-based approach of Morris (1996).

The remainder of the paper is organized as follows: Section 2 presents the framework and the axioms imposed on interpretations and shows how observable preferences are derived. In Section 3, knowledge and belief operators are derived and used to define and characterize parsimony. Sections 4 and 5 relate the representation of observable preferences to knowledge and belief and proposes a compelling form of dynamic consistency for observable preferences.

\section{Framework and axioms}

This section explains how to derive a preference relation representing the DM's observable behavior from axioms defined on the primitive notions of the model. 


\section{$2.1 \quad$ Framework}

The DM is assumed to conform to SEU: he is characterized by a finite state space 2 , a set of outcomes $X$ and a preference relation $\succsim_{\Omega}$ over $\mathcal{A}_{\Omega}$, the set of Savage acts - a Savage act being a function mapping $\Omega$ onto $X$. By SEU, there exist a probability measure $\mathbb{P}$ on $\Omega$ and a real-valued utility function $u$ on $X$ such that: for any acts $F, G \in \mathcal{A}_{\Omega}$, $F \succsim_{\Omega} G \Leftrightarrow \sum_{\omega \in \Omega} \mathbb{P}(\omega) \times u(F(\omega)) \geq \sum_{\omega \in \Omega} \mathbb{P}(\omega) \times u(G(\omega))$. In addition, this framework is enriched by some more primitive notions: a finite set of observations $\mathcal{O}$ and a mapping $\varphi$. A feasible act $f$ is defined as a function mapping $\mathcal{O}$ onto $X$ and the set of such acts is denoted $\mathcal{A}_{\mathcal{O}}$. The mapping $\varphi$ maps each feasible act $f \in \mathcal{A}_{\mathcal{O}}$ onto a Savage act $\varphi(f) \in \mathcal{A}_{\Omega}$.

A subset $E \subseteq \Omega$ is referred to as a Savage event and is said to obtain while a subset $A \subseteq \mathcal{O}$ is referred to as an observable event and is said to be realized. To simplify notations, each outcome $x \in X$ is identified with the constant Savage act $F \in \mathcal{A}_{\Omega}$ defined by $F(\omega)=x$, for all $\omega \in \Omega$, but also with the constant feasible act $f \in \mathcal{A}_{\mathcal{O}}$ defined by $f(o)=x$, for all $o \in \mathcal{O}$. Thus, each $x$ denotes simultaneously the corresponding outcome, constant Savage act and constant feasible act. Moreover, for any two Savage acts $F, G \in \mathcal{A}_{\Omega}$ and any Savage event $E \subseteq \Omega, F_{E} G$ stands for the Savage act equal to $F$ over $E$ and to $G$ over $E^{c}$. The fact that $F$ and $G$ are equal over $E$ is denoted $F==_{E} G$. Similar notations are used for feasible acts. Furthermore, $\Delta(\tilde{\Omega})$ stands for the set of probability measures on a finite set $\tilde{\Omega}$. Finally, for a given subset $\tilde{\Omega} \subseteq \Omega$ and for a feasible act $f \in \mathcal{A}_{\mathcal{O}}$, the notation $\varphi(f) \in \mathcal{A}_{\Omega}$ is also used to denote the restriction of $\varphi(f)$ to $\tilde{\Omega}$.

The fact that probability judgments apply to states while feasible acts are defined over observations creates a problem for the DM: there is no straightforward way to define the probability distribution of a feasible act and therefore it is not clear how feasible acts should be evaluated. The mapping $\varphi$ is precisely the tool used by the DM to overcome this difficulty. Since probability can be used to evaluate Savage acts through the SEU criterion, the DM interprets each feasible act as a Savage act through the mapping $\varphi$ : each outcome $\varphi(f)(\omega)$ stands for the DM's certainty equivalent of $f \in \mathcal{A}_{\mathcal{O}}$ at state $\omega \in \Omega$. As a consequence, the DM chooses a feasible act whose interpretation through $\varphi$ maximizes his expected utility. From now on, the word interpretation refers directly to the mapping $\varphi$.

Definition 1 The observable preference relations $\succsim_{\mathcal{O}}$ are defined by:

$$
\forall f, g \in \mathcal{A}_{\mathcal{O}}, f \succsim_{\mathcal{O}} g \Longleftrightarrow \varphi(f) \succsim_{\Omega} \varphi(g) .
$$

\subsection{Axioms}

The DM is free to use any kind of interpretation provided certain rules are satisfied. These rules take the form of axioms on the mapping $\varphi$.

Axiom $1 \forall f \in \mathcal{A}_{\mathcal{O}}, \forall x \in X$, if $f(o) \sim_{\Omega} x, \forall o \in \mathcal{O}$, then $\varphi(f)(\omega) \sim_{\Omega} x, \forall \omega \in \Omega$.

Axiom $2 \forall o \in \mathcal{O}, \exists \omega \in \Omega$ such that $\forall f \in \mathcal{A}_{\mathcal{O}}, \varphi(f)(\omega) \sim_{\Omega} f(o)$.

\footnotetext{
${ }^{2}$ See Gul (1992) for an axiomatic treatment of SEU over a finite state space.
} 
Axiom $3 \forall \omega \in \Omega, \exists f, g \in \mathcal{A}_{\mathcal{O}}$ such that $\varphi(f)(\omega) \succ_{\Omega} \varphi(g)(\omega)$.

Let $\Omega(\mathcal{O}) \subseteq \Omega$ stands for the set of states involved by $\mathbf{A} \mathbf{2}$.

Axiom $4 \forall \Omega^{\prime} \subseteq \Omega$ with $\Omega(\mathcal{O}) \subseteq \Omega^{\prime}$, if $\exists\left(u^{\prime}, \mathbb{P}^{\prime}\right) \in \mathbb{R}^{X} \times \Delta\left(\Omega^{\prime}\right)$ such that $\forall f, g \in \mathcal{A}_{\mathcal{O}}$, $\mathbb{E}_{\mathbb{P}} u(\varphi(f)) \geq \mathbb{E}_{\mathbb{P}} u(\varphi(g)) \Longleftrightarrow \mathbb{E}_{\mathbb{P}^{\prime}} u^{\prime}(\varphi(f)) \geq \mathbb{E}_{\mathbb{P}}^{\prime} u^{\prime}(\varphi(g))$, then $\Omega^{\prime}=\Omega$.

Under A1, each feasible act inducing no uncertainty is interpreted as a Savage act inducing no uncertainty. ${ }^{3}$ Moreover, their utilities are the same. Put differently, the process of interpretation only becomes necessary in situations of uncertainty. Under A2, for each observation, there exists a state of the world such that the DM behaves at that state as if that observation was necessarily realized. In other words, this axiom forces all nonambiguous states to belong to the state space which appears to be an extension of the set of observations. Besides, A3 is a nontriviality requirement: each state encodes at least one possible observation. Since all states in $\Omega(\mathcal{O})$ encode a single observation - i.e. they are nonambiguous - , then all states in $\Omega \backslash \Omega(\mathcal{O})$ are ambiguous and possibly encode more than one observation. Finally, A4 optimizes the way $\Omega$ extends $\mathcal{O}: \Omega$ must be a minimal state space to generate observable preferences.

The two following examples illustrate interpretations that satisfy the above axioms.

Example 1 - A simple case of interpretation is given by $\Omega=\mathcal{O}$ and $\varphi=I d$. This situation captures the idea that the DM has a perfect knowledge of states: at each possible state, he knows the observation for sure. Hence, each possible state fully determines the observation. In this case, observable behavior is directly represented by preferences $\succsim_{\Omega}$ and is therefore SEU.

Example 2 - Another particular case already appearing in the literature (Gilboa and Schmeidler, 1994; Mukerji, 1997) is given by $\Omega=2^{\mathcal{O}}$ and $u(\varphi(f)(\omega))=\min _{o \in \omega} u(f(o))$, for all feasible act $f \in \mathcal{A}_{\mathcal{O}}$ and $\omega \in 2^{\mathcal{O}}$. This specification translates some imperfection in knowledge of states. However, such imperfections end up generating observable behavior of a very specific type, namely it can only be CEU wrt a belief function.

\section{Knowledge and parsimony}

This section begins with an example meant to provide intuitions on knowledge and parsimony which are then defined more formally in terms of the primitives of the model.

\footnotetext{
${ }^{3}$ In $\mathrm{A} 1, f(o)$ stands for the Savage act that maps each state onto the consequence $f(o)$.
} 


\subsection{State ambiguity in the Ellsberg urn}

In Ellsberg (1961), an urn is assumed to contain 90 balls, 30 of which are red $(r)$ while the remaining ones are blue $(b)$ or white $(w)$ in unknown proportions (for clarity, observations are written with small-letters, while states are written with capital-ones). A ball is about to be drawn in the urn and the DM must choose between a 'bet on red' and a 'bet on blue' in situation 1 and between a 'bet on not-blue' and a 'bet on not-red' in situation 2. The corresponding outcomes are given in the tables hereafter.

\begin{tabular}{cccccccc}
\hline situation 1 & $r$ & $b$ & $w$ & situation 2 & $r$ & $b$ & $w$ \\
\hline$\underline{\text { bet on } r}$ & 1 & 0 & 0 & bet on $r^{c}$ & 1 & 0 & 1 \\
\hline bet on $b$ & 0 & 1 & 0 & $\underline{\text { bet on } b^{c}}$ & 0 & 1 & 1 \\
\hline
\end{tabular}

Observable behavior in the Ellsberg experiment is taken to consist of the choices underlined in the tables, which are referred to as the Ellsberg choices.

Standard decision-theoretic practice describes uncertainty by means of a state space where each state is considered as nothing but a possible observation. As explained in the introduction, this precludes the possibility of unforeseen contingencies and failures of logical omniscience. Consider now the following version of the Ellsberg urn:

(1) each ball is characterized by two numbers $(i, j)$ with $i \in\{0,1,2,3\}$ and $j \in\{0,1\}$, and

(2) these numbers fully work out the colour of the ball according to the following rules:

\begin{tabular}{ccccc}
\hline & $i=0$ & $i=1$ & $i=2$ & $i=3$ \\
\hline$j=0$ & $r$ & $b$ & $w$ & $b$ \\
\hline$j=1$ & $r$ & $b$ & $w$ & $w$ \\
\hline
\end{tabular}

Suppose that the DM understands the importance of $i$ but fails to see the relevance of $j$. Then, his representation of the uncertainty attached to the color of a ball is given by the following table:

\begin{tabular}{cccc}
\hline$i=0$ & $i=1$ & $i=2$ & $i=3$ \\
\hline$r$ & $b$ & $w$ & $\{b, w\}$ \\
\hline
\end{tabular}

This situation can be modeled through a state space $\Omega=\{R, B, W, \Psi\}$ where each state corresponds to one of the values of $i$; in particular, $\Psi$ to $i=3$. Hence the DM perceives a form of ambiguity at $\Psi$ which is from now on referred to as state ambiguity. At this ambiguous state, he knows that $\{b, w\}$ is realized without knowing whether $\{b\}$ is realized or $\{w\}$ is realized.

The following specification of the interpretation $\varphi$ reflects faithfully state ambiguity in the Ellsberg urn above. Fix a utility function $u: X \rightarrow R$ and, for all $f \in \mathcal{A}_{\mathcal{O}}$, let:

$$
\left\{\begin{array}{l}
\varphi(f)(R)=u(f(r)), \\
\varphi(f)(B)=u(f(b)), \\
\varphi(f)(W)=u(f(w)), \\
\varphi(f)(\Psi)=E_{\nu_{\Psi}} u(f),
\end{array}\right.
$$


where $\nu_{\Psi}$ is a Choquet capacity over $\mathcal{O}$ defined by $\nu_{\Psi}(\varnothing)=\nu_{\Psi}(\{r\})=0, \nu_{\Psi}(\{b\})=$ $\nu_{\Psi}(\{r, b\})=p, \nu_{\Psi}(\{w\})=\nu_{\Psi}(\{r, w\})=q$, with $\left.p, q \in\right] 0,1\left[\right.$, and $\nu_{\Psi}(\{b, w\})=\nu_{\Psi}(\{r, b, w\})=$ 1 , and the expectation refers to the Choquet integral (Schmeidler, 1989). Note that, contrary to the logic of Möbius transform (Shafer, 1976), the capacity $\nu_{\Psi}$ is not required to be a unanimity game.

\subsection{Knowledge}

The Ellsberg urn example suggests that state ambiguity puts the light on the DM's knowledge. The interpretation $\varphi$ is now used to derive formally a knowledge operator in the following way. At each state $\omega \in \Omega$ where the DM can tell precisely the observation $o \in \mathcal{O}$ which is realized, he is expected to be indifferent between any feasible act $f \in \mathcal{A}_{\mathcal{O}}$ and outcome $f(o)$. When this happens, the DM is said to know o at $\omega$. More generally, assume that the DM knows observable event $A \subseteq \mathcal{O}$ at some state. He is then expected to be indifferent at that state between any two acts whose outcomes are equal over $A$. Such a preference-based approach to knowledge follows the spirit of Morris' (1996) construction, as opposed to Aumann's (1999) seminal one which rather relies on syntactic modalities.

Definition 2 For each $A \subseteq \mathcal{O}$, let:

$$
\left.K_{\Omega}(A)=\left\{\omega \in \Omega, \forall f, g \in \mathcal{A}(\mathcal{O}), f={ }_{A} g \Longrightarrow \varphi(f)(\omega) \sim_{\Omega} \varphi(g)(\omega)\right\}\right\} .
$$

The expression $K_{\Omega}(A)$ is here directly interpreted as 'the subset of states at which the DM considers that $A$ is necessarily realized' - in short 'the DM knows $A$ is realized'. Whereas, the expression $K_{\Omega}\left(A^{c}\right)^{c}$ is interpreted as 'the subset of states at which the DM does not considers that $A^{c}$ cannot be realized'-in short 'the DM believes $A$ is realized':

$$
B_{\Omega}(A)=K_{\Omega}\left(A^{c}\right)^{c}
$$

The DM knows (resp. believes) exactly what he considers as necessary (resp. possible). Thus, knowledge offers a specific way to represent observations as Savage events.

Example 1 (cont.) - When the interpretation $\varphi$ is given by the identity function, Definition 2 implies the following form of knowledge: for all $A \subseteq \mathcal{O}, K_{\Omega}(A)=B_{\Omega}(A)=$ $\{\omega \in \Omega, \omega \in A\}$.

Example 2 (cont.) — Under a specification à la Gilboa and Schmeidler (1994) and Mukerji (1997), Definition 2 implies the following form of knowledge: for all $A \subseteq \mathcal{O}$, $K_{\Omega}(A)=\{\omega \in \Omega, \omega \subseteq A\}$ and $B_{\Omega}(A)=\{\omega \in \Omega, \omega \cap A \neq \varnothing\}$. In Mukerji's terminology, operator $K_{\Omega}$ is called the inverse implication mapping.

An observable event $A \subseteq \mathcal{O}$ that is not affected by state ambiguity is such that one of $A$ or $A^{c}$ is known at each state. The family $\left(K_{\Omega}(A), B_{\Omega}\left(A^{c}\right)\right)$ defines therefore a partition over $\Omega$ which amounts to an agreement of knowledge and belief on $A$. Such an event $A$ is called a measurable observable event. 
Definition 3 An observable event $A \subseteq \mathcal{O}$ is said to be measurable if

$$
K_{\Omega}(A)=B_{\Omega}(A) .
$$

Let $\mathcal{M}_{\mathcal{O}}$ and $\mathcal{A}_{\mathcal{O}}^{\mathcal{M}}$ stand respectively for the sets of measurable observable events and measurable feasible acts.

In the Ellsberg urn described by the mapping $\varphi$ used in Section 3.1, knowledge and belief are given by the following table:

\begin{tabular}{lcccccc}
\hline$A$ & $\{r\}$ & $\{b\}$ & $\{w\}$ & $\{r, b\}$ & $\{r, w\}$ & $\{b, w\}$ \\
\hline$K_{\Omega}(A)$ & $\{R\}$ & $\{B\}$ & $\{W\}$ & $\{R, B\}$ & $\{R, W\}$ & $\{B, W, \Psi\}$ \\
\hline$B_{\Omega}(A)$ & $\{R\}$ & $\{B, \Psi\}$ & $\{W, \Psi\}$ & $\{R, B, \Psi\}$ & $\{R, W, \Psi\}$ & $\{B, W, \Psi\}$ \\
\hline
\end{tabular}

The set of measurable observable events can be read directly in the table and is equal to $\mathcal{M}_{\mathcal{O}}=\{\varnothing,\{r\},\{b, w\}, \mathcal{O}\}$.

\subsection{Parsimony}

As shown by the Ellsberg Urn example in the above section, knowledge and belief can disagree and thus generate nonmeasurable observable events. However, the DM is said to conform to parsimony when he spares himself the distinction between observability and measurability. This means that the DM does not perceive state ambiguity and that his knowledge and belief necessarily agree.

Definition 4 The DM conforms to parsimony if any observable event is measurable.

Proposition 1 characterizes parsimony in terms of the primitives of the model in making the connection between the formal definition of parsimony and the identification of states of the world with observations.

Proposition 1 The DM conforms to parsimony if and only if, for each state $\omega \in \Omega$, there exists an observation $o \in \mathcal{O}$ such that: $\forall f \in \mathcal{A}_{\mathcal{O}}, \varphi(f)(\omega) \sim_{\Omega} f(o)$.

Proof. $(\longleftarrow)$ Assume the characterizing condition. To show parsimony, it is sufficient to prove $B(A) \subseteq K(A)$, for all $A \subseteq \mathcal{O}$. Consider a state $\omega \in B(A)$. Then, by definition, there exist feasible acts $f, g \in \mathcal{A}_{\mathcal{O}}$ that are equal over $A^{c}$ and satisfy $\varphi(f)(\omega) \succ_{\Omega} \varphi(g)(\omega)$. Let $o \in \mathcal{O}$ be an observation corresponding to $\omega$ as in the characterizing condition. Then, $f(o) \succ_{\Omega} g(o)$ and therefore $o$ has to belong to $A$. But then, take any two feasible acts $f, g \in \mathcal{A}_{\mathcal{O}}$ that are equal over $A$. One has $f(o) \sim_{\Omega} g(o)$ and necessarily $\varphi(f)(\omega) \sim_{\Omega} \varphi(g)(\omega)$ so that $\omega \in K(A)$. Hence parsimony. (Intermediate) For the reciprocal implication, define, for each state $\omega$, the subset $n(\omega) \subseteq \mathcal{O}$ as the intersection of all observable events $A \subseteq \mathcal{O}$ such that $\omega \in K(A)$. Note that $K$ preserves finite intersections. Indeed, it is easy to see that $K$ is monotonic and therefore, for any $A, B \subseteq \mathcal{O}, K(A \cap B) \subseteq K(A) \cap K(B)$. In addition, assume $\omega \in K(A) \cap K(B)$. Consider two acts $f, g \in \mathcal{A}_{\mathcal{O}}$ such that $f={ }_{A \cap B} g$. 
Then, there is a feasible act $h \in \mathcal{A}_{\mathcal{O}}$ such that $g=f_{A \cap B} h=\left(f_{B} h\right)_{A} h$. Since $\omega \in K(A)$, $\varphi(g)(\omega) \sim_{\Omega} \varphi\left(f_{B} h\right)(\omega)$ and, since $\omega \in K(B), \varphi\left(f_{B} h\right)(\omega) \sim_{\Omega} \varphi(f)(\omega)$. By transitivity, $\varphi(g)(\omega) \sim_{\Omega} \varphi(f)(\omega)$ and $\omega \in K(A \cap B)$. Therefore, since $\mathcal{O}$ is finite, it is true that, for any state $\omega \in \Omega, \omega \in K(n(\omega))$. In addition, by $\mathbf{A} 3, n(\omega)$ is never empty. ( $\longrightarrow)$ Assume now parsimony. Let us first show that each $n(\omega)$ is a singleton. Let $o, o^{\prime} \in n_{\mathcal{O}}(\omega)$ and $\omega^{\prime}$ correspond to $o^{\prime}$ as in A2. Necessarily then, $\omega^{\prime} \in K\left(\left\{o^{\prime}\right\}\right)$ and $n\left(\omega^{\prime}\right) \subseteq\left\{o^{\prime}\right\}$. Since $n\left(\omega^{\prime}\right)$ cannot be empty, it follows that $n\left(\omega^{\prime}\right)=\left\{o^{\prime}\right\}$. If $\omega \in K\left(\left\{o^{\prime}\right\}^{c}\right)$, then $o^{\prime} \in n(\omega) \subseteq\left\{o^{\prime}\right\}^{c}$, which is absurd. Therefore, $\omega \in B\left(\left\{o^{\prime}\right\}\right)$ and, by parsimony, $\omega \in K\left(\left\{o^{\prime}\right\}\right)$. Finally, $o \in$ $n(\omega) \subseteq\left\{o^{\prime}\right\}$ and $o=o^{\prime}$. Each $n(\omega)$ is indeed a singleton. For each $\omega \in \Omega$, let $o \in O$ be such that $n_{\mathcal{O}}(\omega)=\{o\}$. Fix $f \in A_{\mathcal{O}}$ to show $\varphi(f)(\omega) \sim_{\Omega} f(o)$. Let $x=f(o) \in X$ so that $f=x$ over $\{o\}$. Then, $\varphi(f)(\omega) \sim_{\Omega} \varphi(x)(\omega)$. Using A1, it follows that $\varphi(f)(\omega) \sim_{\Omega} x \sim_{\Omega} f(o)$.

Proposition 1 shows that parsimony is characterized by the existence of a function $\epsilon$ mapping states onto observations such that, at each state $\omega \in \Omega$, the DM knows $\epsilon(\omega)$. Under parsimony, then, each possible state fully determines the observation. Hence, Example 1 provides a typical case of parsimony where $\Omega=\mathcal{O}$ and $\varphi=I d$.

\section{Representation of observable preferences}

This section studies the representation of observable preferences with and without parsimony, i.e. when knowledge and belief agree or disagree.

\subsection{SEU representation}

The next proposition shows that, if knowledge and belief agree, then the DM's observable preferences are SEU wrt the set $\mathcal{O}$ of observations. Therefore, the DM assesses the likelihood of all observable events through a standard additive probability measure.

Proposition 2 The DM conforms to parsimony if and only if his observable preferences are SEU.

Proof. $(\longrightarrow)$ Assume parsimony. Given Definition 1, observable preferences can be represented through the functional $V: \mathcal{A}_{\mathcal{O}} \rightarrow \mathbb{R}$ defined, for any feasible act $f \in \mathcal{A}_{\mathcal{O}}$, by $V(f)=\mathbb{E}_{\mathbb{P}} u(\varphi(f))$. Under parsimony, each subset $n(\omega) \subseteq \mathcal{O}$ (see its definition in the above proof of Proposition 1) is a singleton. For each $\omega \in \Omega$, let $\epsilon(\omega) \in \mathcal{O}$ be the observation defined by $n(\omega)=\{\epsilon(\omega)\}$ and note that $u(\varphi(f)(\omega))=u(f(\epsilon(\omega)))$. Then, $V(f)=\mathbb{E}_{P} u(f)$ where $P$ is the probability distribution over $\mathcal{O}$ induced by $\epsilon$ under $\mathbb{P}$ and observable preferences are indeed SEU. $(\longleftarrow)$ Assume now the existence of a utility function $v$ and a probability measure $P$ over $\mathcal{O}$ such that the functional $V: \mathcal{A}_{\mathcal{O}} \rightarrow \mathbb{R}$ defined, for any feasible act $f \in \mathcal{A}_{\mathcal{O}}$, by $V(f)=\mathbb{E}_{P} v(f)$ represents observable preferences $\succsim_{\mathcal{O}}$. To show parsimony, take $\Omega^{\prime}=\Omega(\mathcal{O})$ and construct $u^{\prime} \in \mathbb{R}^{X}$ and $\mathbb{P}^{\prime} \in \Delta\left(\Omega^{\prime}\right)$ as in A4. Let $u^{\prime}=v$ and use $\mathbf{A} 3$ to define $\mathbb{P}^{\prime}$ according to $\mathbb{P}^{\prime}(\{\omega\})=P(\{o\})$ where, for each state $\omega \in \Omega$, the observation $o \in \mathcal{O}$ corresponds to $\omega$ as in A2. Then, for any feasible act 
$f \in \mathcal{A}_{\mathcal{O}}, V(f)=\mathbb{E}_{\mathbb{P}^{\prime}} u^{\prime}(\varphi(f))$. Finally, A4 implies that $\Omega=\Omega^{\prime}=\Omega(\mathcal{O})$, which amounts to parsimony.

As a corollary, departures from SEU and, in particular, sensitivity to Knightian uncertainty (Knight, 1921) here called classical ambiguity, reveal that knowledge and belief disagree: there are ambiguous states at which the DM considers that some event is possible but does not know this event for sure. Therefore, this approach leads to a seemingly new interpretation of the Ellsberg choices in terms of state ambiguity.

The next theorem shows that, even when the DM perceives some state ambiguity, he can still be said to conform to parsimony, at least over a limited subdomain of feasible acts. More precisely, Theorem 1 delivers a SEU representation of the restriction of observable preferences to measurable feasible acts.

Theorem 1 The following assertions hold:

(1) $\forall A \in \mathcal{M}_{\mathcal{O}}, P(A)=\mathbb{P}\left[K_{\Omega}(A)\right]$ defines a probability measure over $\left(\mathcal{O}, \mathcal{M}_{\mathcal{O}}\right)$.

(2) $\forall f, g \in \mathcal{A}_{\mathcal{O}}^{\mathcal{M}}, f \succsim_{\mathcal{O}} g \Longleftrightarrow \mathbb{E}_{P} u(f) \geq \mathbb{E}_{P} u(g)$.

Proof. (1) Note that $K_{\Omega}$ preserves intersections while $B_{\Omega}$ preserves unions. Their common restriction to $\mathcal{M}_{\mathcal{O}}$ therefore preserves both operations and the set function $P$ is indeed a probability measure over $\mathcal{O}$. (2). Consider now a measurable feasible act $f \in \mathcal{A}_{\mathcal{O}}^{\mathcal{M}}$. It is of the form: $f=\sum_{i=1}^{n} \mathbb{1}_{A_{i}} x_{i}$ with all $x_{i}$ pairwise distinct and all $A_{i}$ measurable. By A1 and since $f=x_{i}$ over $A_{i}, u(\varphi(f)(\omega))=u\left(\varphi\left(x_{i}\right)(\omega)\right)=u\left(x_{i}\right)$, for all $\omega \in \mathbf{K}_{\Omega}\left(A_{i}\right)$. Moreover, the family $\left(K_{\Omega}\left(A_{i}\right)\right)_{i=1}^{n}$ is a partition of $\Omega$. Then, the expected utility induced by $\varphi(f)$ can be written in the following way:

$$
\mathbb{E}_{\mathbb{P}} u(\varphi(f))=\sum_{i=1}^{n} u\left(x_{i}\right) \mathbb{P}\left[K_{\Omega}\left(A_{i}\right)\right]=\mathbb{E}_{P} u(f) .
$$

Theorem 1.2 then follows from the definition of observable preferences and the SEU representation of Savage preferences.

Technically, Theorem 1 generalizes Proposition 2: under parsimony, the algebra $\mathcal{M}_{\mathcal{O}}$ of measurability becomes equal to the powerset of $\mathcal{O}$ and, therefore, sEU is implied over the whole domain of feasible acts. By its simple definition, $\mathcal{M}_{\mathcal{O}}$ is a state ambiguity-free domain in the sense that each measurable observable event is either necessarily realized or impossible at any state. Theorem 1 shows also that $\mathcal{M}_{\mathcal{O}}$ is a classical ambiguity-free domain in the sense that the DM assigns a unique probability to each measurable observable event. In addition, part (1) of the theorem explicitly relates probability assessments to the DM's knowledge: the probability of event $A \in \mathcal{M}_{\mathcal{O}}$ is to be understood as the probability of being in some of the states at which $A$ is known. Finally, while sEU is considered as the reference model of full rationality under uncertainty (Al-Najjar and Weinstein, 2009), Theorem 1 suggests that the disagreement between knowledge and belief is a possible characterization of bounded rationality. In this respect, the size of the algebra $\mathcal{M}_{\mathcal{O}}$ would measure the degree of rationality of the individual and therefore lead to a comparative notion of rationality. 


\subsection{CEU representation}

Contrary to Proposition 1 and Theorem 1, the next theorem dispenses with parsimony and gives a functional representation of observable preferences over the full set $\mathcal{A}_{\mathcal{O}}$ of feasible acts. It also derives a second representation in which observable preferences are CEU. This result could easily be adapted to the case of Maxmin Expected Utility (MEU) preferences (Gilboa and Schmeidler, 1989). While Proposition 2 gives rise to the intuition that classical ambiguity can always be reduced to state ambiguity, Theorem 2 further explains that observable preferences under state ambiguity replicate standard models of classical ambiguity.

In Theorem 2, the Choquet case refers to interpretations $\varphi$ designed in the following way: $\forall f \in \mathcal{A}_{\mathcal{O}}, u(\varphi(f)(\omega))=\mathbb{E}_{\nu_{\omega}} u(f)$, where, for each $\omega \in \Omega, \nu_{\omega}$ is a capacity over $\mathcal{O}$ as in the CEU model of Schmeidler (1989). Remark that state ambiguity in the Ellsberg urn as described in Section 3.1 is a typical illustration of the Choquet case.

Theorem 2 Assume that Savage preferences $\succsim_{\Omega}$ are SEU wrt some utility function $u$ and some probability measure $\mathbb{P}$ over $\Omega$.

(1) $\forall f, g \in \mathcal{A}_{\mathcal{O}}, f \succsim \mathcal{O} g \Longleftrightarrow \mathbb{E}_{\mathbb{P}} u(\varphi(f)) \geq \mathbb{E}_{\mathbb{P}} u(\varphi(g))$.

Moreover, in the Choquet case:

(2) $\succsim_{\mathcal{O}}$ is $\mathrm{CEU}$ wrt $u$ and capacity $\nu_{0}$ defined by $\nu_{0}(A)=\sum_{\omega \in \Omega} \nu_{\omega}(A) \mathbb{P}[\{\omega\}], \forall A \subseteq \mathcal{O}$,

(3) $\forall A \subseteq \mathcal{O}, \mathbb{P}\left[K_{\Omega}(A)\right] \leq \nu_{0}(A) \leq \mathbb{P}\left[B_{\Omega}(A)\right]$.

Proof. (1) is obtained through Definition 1 and the SEU assumption for Savage preferences. (2) follows from the equality $\mathbb{E}_{\mathbb{P}} u(\varphi(f))=\mathbb{E}_{\nu_{0}} u(f)$, for all feasible act $f \in \mathcal{A}_{\mathcal{O}}$. (3) If $\omega \in K_{\Omega}(A)$, then $x_{A} y \sim_{\omega} x$, for all $x, y \in X$ and $\nu_{\omega}(A)=1$. Similarly, if $\omega \notin B_{\Omega}(A)$, then $x_{A} y \sim_{\omega} y$, for all $x, y \in X$ and $\nu_{\omega}(A)=0$.

The representation described in Theorem 2.1 is a straightforward consequence of the definition of observable preferences and the SEU assumption imposed on $\succsim \Omega$. Second, in the Choquet case where the interpretation is given through a family $\left(\nu_{\omega}\right)_{\omega \in \Omega}$, observable preferences are CEU wrt a capacity $\nu_{0}$ obtained as the mathematical expectation under $\mathbb{P}$ of capacities $\nu_{\omega}$. This definition of $\nu_{0}$ is reminiscent of the logic of Möbius inversion (Dempster, 1967) and its decision-theoretic implementation (Mukerji, 1997). In these contributions, the family $\left(\nu_{\omega}\right)_{\omega \in \Omega}$ degenerates into the family of unanimity games on $\mathcal{O}$ and therefore $\nu_{0}$ is a belief function. Finally, Theorem 2.3 imposes a consistency requirement between the capacity $\nu_{0}$ revealed by observable preferences and the epistemic operators of knowledge and belief implied by the interpretation $\varphi$ : the two operators impose bounds on the degree of ambiguity aversion (Ghirardato and Marinacci, 2002) revealed by observable preferences. Note that the lower bound $\mathbb{P}\left[K_{\Omega}().\right]$ can be shown to be a belief function while the upper bound $\mathbb{P}\left[B_{\Omega}().\right]$ corresponds to the dual plausibility function (Demspter, 1967). Consistently with Theorem 1 , these two bounds naturally coincide over the algebra of measurability. This finally reveals that classical ambiguity is state ambiguity. 


\section{Dynamics}

This section develops a form of dynamic consistency that integrates state ambiguity and remains achievable under classical ambiguity. This form of dynamic consistency is then illustrated through the Ellsberg example.

\subsection{Dynamic consistency}

Models of classical ambiguity rationalize the Ellsberg choices interpreted as violations of SEU: parsimony is typically maintained while the sure Thing Principle (STP) and dynamic consistency are typically lost. Here, the Ellsberg choices are interpreted as revealing state ambiguity and lead to an alternative rationalization in which parsimony is violated while dynamic consistency is always preserved. This form of dynamic consistency can be reformulated over the set $\mathcal{O}$ of observations. Without parsimony, that is when knowledge and belief disagree, dynamic consistency involves two families of preferences: $\left(\succsim_{A}^{K}\right)_{A \subseteq \mathcal{O}}$ and $\left(\succsim_{A}^{B}\right)_{A \subseteq \mathcal{O}}$.

Theorem 3 There exist two families of preference relations over feasible acts, denoted $\left(\succsim_{A}^{K}\right)_{A \subseteq \mathcal{O}}$ and $\left(\succsim_{A}^{B}\right)_{A \subseteq \mathcal{O}}$, such that for all $A \subset \mathcal{O}$ :

(1) $\forall f, g \in \mathcal{A}_{\mathcal{O}}, f \succsim_{A}^{K} g$ and $f \succsim_{A^{c}}^{B} g \Longrightarrow f \succsim_{\mathcal{O}} g$.

(2) $\forall f, g \in \mathcal{A}_{\mathcal{O}}, \forall \omega \in K_{\Omega}(A), \varphi(f)(\omega) \succsim_{\Omega} \varphi(g)(\omega) \Longrightarrow f \succsim_{A}^{K} g$.

(3) $\forall f, g \in \mathcal{A}_{\mathcal{O}}, \forall \omega \in B_{\Omega}(A), \varphi(f)(\omega) \succsim_{\Omega} \varphi(g)(\omega) \Longrightarrow f \succsim_{A}^{B} g$.

Proof. Given the SEU assumption imposed on $\succsim_{\Omega}$, it is possible to define a family $\left(\succsim_{E}\right)_{E \subseteq \Omega}$ of preferences over Savage acts in the following way: for all $E \subseteq \Omega$ and all $F, G \in \mathcal{A}_{\Omega}, F \succsim_{E} G \Longleftrightarrow \mathbb{E}_{\mathbb{P}}[u(F) \mid E] \geq \mathbb{E}_{\mathbb{P}}[u(G) \mid E]$, if $\mathbb{P}(E)>0$, and $F \sim_{E} G$ otherwise. Then, the two classical conditions hereafter hold for all $E \subseteq \Omega$ :

$$
\left\{\begin{array}{l}
\text { (a) } \forall F, G \in \mathcal{A}_{\Omega}, F \succsim_{E} G \text { and } F \succsim_{E^{c}} G \Longrightarrow F \succsim_{\Omega} G, \\
\text { (b) } \forall F, G \in \mathcal{A}_{\Omega},\left(\forall \omega \in E, F(\omega) \succsim_{\Omega} G(\omega)\right) \Longrightarrow F \succsim_{E} G .
\end{array}\right.
$$

Ex post preferences over feasible acts can then be defined by: $\forall E \subseteq \Omega, \forall f, g \in \mathcal{A}_{\mathcal{O}}$, $f \succsim_{E} g \Longleftrightarrow \varphi(f) \succsim_{E} \varphi(g)$. In addition, let the preferences in the families $\left(\succsim_{A}^{K}\right)_{A \subseteq \mathcal{O}}$ and $\left(\succsim_{A}^{B}\right)_{A \subseteq \mathcal{O}}$ be defined for each observable event $A \subseteq \mathcal{O}$ by:

$$
\left\{\begin{array}{l}
(\alpha) \forall f, g \in \mathcal{A}_{\mathcal{O}}, f \succsim_{A}^{K} g \Longleftrightarrow f \succsim_{K_{\Omega}(A)} g \\
(\beta) \forall f, g \in \mathcal{A}_{\mathcal{O}}, f \succsim_{A}^{B} g \Longleftrightarrow f \succsim_{B_{\Omega}(A)} g
\end{array}\right.
$$

If $A$ is measurable, then $K_{\Omega}(A)=B_{\Omega}(A)$ and preferences $\succsim_{A}^{K}$ and $\succsim_{A}^{B}$ coincide. At last, conditions (1), (2) and (3) directly follow from properties (a) and (b) above together with definitions $(\alpha)$ and $(\beta)$.

Conditional upon knowing (resp. believing) observable event $A$, the DM maximizes $\succsim_{A}^{K}$ (resp. $\left.\succsim_{A}^{B}\right)$ subject to feasibility constraints. Theorem 3.1 generalizes the classical 
form of dynamic consistency (see Machina, 1989; Ghirardato, 2002) to the present framework of state ambiguity while Theorem 3.2 and Theorem 3.3 extend the expression of consequentialism (see Hammond, 1988). Consequentialism requires behavior to depend upon information and feasibility constraints only. In this respect, consequentialism is still preserved here if one accepts the idea that information now consists, not only of some observable event, but also of one of the two modalities, knowledge or belief, through which the observable event is processed. In addition, it is straightforward to show that $\succsim_{A}^{K}$ and $\succsim_{A}^{B}$ coincide whenever observable event $A$ is measurable. Then, under parsimony, dynamic consistency and consequentialism as captured by Theorem 3 degenerate into their classical version.

Furthermore, dynamic consistency in Theorem 3 can be used to produce updating rules. In the Choquet case studied in Theorem $2, \succsim_{\mathcal{O}}, \succsim_{A}^{K}$ and $\succsim_{A}^{B}$, for all $A \subseteq \mathcal{O}$, are also CEU wrt $u$ and capacities $\nu_{0}, \nu_{A}^{K}$ and $\nu_{A}^{B}$ defined, for all observable events $A, A^{\prime} \subseteq \mathcal{O}$ such that $\mathbb{P}\left[K_{\Omega}(A)\right]>0$, by:

$$
\left\{\begin{array}{l}
\nu_{0}\left(A^{\prime}\right)=\sum_{\omega \in \Omega} \nu_{\omega}\left(A^{\prime}\right) \mathbb{P}[\{\omega\}], \\
\nu_{A}^{K}\left(A^{\prime}\right)=\sum_{\omega \in \Omega} \nu_{\omega}\left(A^{\prime}\right) \mathbb{P}\left[\{\omega\} \mid K_{\Omega}(A)\right], \\
\nu_{A}^{B}\left(A^{\prime}\right)=\sum_{\omega \in \Omega} \nu_{\omega}\left(A^{\prime}\right) \mathbb{P}\left[\{\omega\} \mid B_{\Omega}(A)\right] .
\end{array}\right.
$$

\section{$5.2 \quad$ Application to Ellsberg choices}

Back to the example of state ambiguity in the Ellsberg urn and using the specification given in Section 3.1, the table given hereafter computes $\nu_{0}, \nu_{\{r, b\}}^{K}$ and $\nu_{\{r, b\}}^{B}$ under the additional assumption that $\mathbb{P}(\{R\})=\mathbb{P}(\{\Psi\})=1 / 3$ and $\mathbb{P}(\{B\})=\mathbb{P}(\{W\})=1 / 6$ :

\begin{tabular}{lcccccc}
\hline$A$ & $\{r\}$ & $\{b\}$ & $\{w\}$ & $\{r, b\}$ & $\{r, w\}$ & $\{b, w\}$ \\
\hline$\nu_{0}(A)$ & $\frac{1}{3}$ & $\frac{1}{6}+\frac{1}{3} p$ & $\frac{1}{6}+\frac{1}{3} q$ & $\frac{1}{2}+\frac{1}{3} p$ & $\frac{1}{2}+\frac{1}{3} q$ & $\frac{2}{3}$ \\
\hline$\nu_{\{r, b\}}^{K}(A)$ & $\frac{2}{3}$ & $\frac{1}{3}$ & 0 & 1 & $\frac{2}{3}$ & $\frac{1}{3}$ \\
\hline$\nu_{\{r, b\}}^{B}(A)$ & $\frac{2}{5}$ & $\frac{1}{5}+\frac{2}{5} p$ & $\frac{2}{5} q$ & $\frac{3}{5}+\frac{2}{5} p$ & $\frac{2}{5}+\frac{2}{5} q$ & $\frac{3}{5}$ \\
\hline
\end{tabular}

Assume that the DM follows the Ellsberg choices. It must be the case that $p<\frac{1}{2}$ and $q<\frac{1}{2}$. The model is now used to construct a dynamically consistent extension of these Ellsberg choices. Consider the following four decision trees.

In the two upper trees, either the DM knows the ball to be red or blue or he believes the ball to be white. The underlying partition over $\Omega$ is therefore given by $(\{R, B\},\{W, \Psi\})$. Similarly, in the two lower trees, either the DM believes the ball to be red or blue or he knows the ball to be white and the underlying partition is given by $(\{R, B, \Psi\},\{W\})$. In the left hand side trees, feasible acts are the same and consist of a 'bet on red' and a 'bet on blue' while, in the right hand side trees, feasible acts are again the same and involve a 'bet on not-red' and a 'bet on not-blue'.

In the two upper trees, choices at decision nodes are determined by calculus. For instance, in the upper left tree, the table above makes it clear that $\nu_{\{r, b\}}^{K}$ assigns more weight on $\{r\}$ than on $\{b\}$. Hence the choice to bet on red. In the two lower trees, choices 

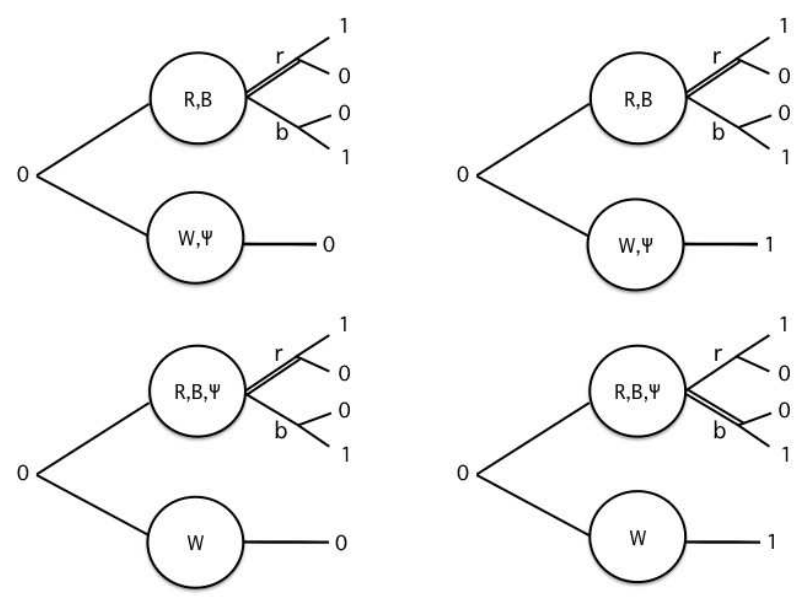

are rather determined by application of consequentialism and dynamic consistency. In the lower left tree, the two acts are indifferent at each state at which the observation $w$ is known. By consequentialism, they are indifferent at $K(\{w\})=\{W\}$. Then, by dynamic consistency, choices are fully determined by ex ante preferences: the choice to bet on red follows directly from the Ellsberg pattern of choice. As indicated in the above figure, the precise 'location' of the ambiguous state $\Psi$ at upper or lower decision node affects choices.

The standard parsimonious dynamic extension of the Ellsberg choices is known to yield a paradoxical situation: choices conditional upon a particular event depends on counterfactual outcomes (see Al-Najjar and Weinstein, 2009, Section 2.4). However, in a nonparsimonious setting, the paradoxical situation is solved through state ambiguity. For instance, in the above lower trees, the presence of ambiguous state $\Psi$ makes the observation $w$ possible at upper nodes. Therefore, the associated outcome is no more counterfactual and the fact it affects behavior is no longer paradoxical.

Finally, the Ellsberg choices and other departures from SEU are here interpreted as departures from parsimony and therefore lead to state ambiguity and disagreement between knowledge and belief. Once this disagreement is taken into account in the dynamic extension of behavior, it becomes possible to construct a form of dynamic consistency that is adequate for non-SEU preferences.

\section{References}

Al-NajJar, N.I. And J.L. Weinstein (2009). 'The Ambiguity Aversion Literature: A Critical Assessment,' Economics and Philosophy 25, 249-284.

Aumann, R.J. (1999). 'Interactive epistemology I: Knowledge,' International Journal of Game Theory 28, 263-300.

Dekel, E., Lipman, B.L. And A. Rustichini (2001). 'Representing Preferences with a Unique Subjective State Space,' Econometrica 69, 891-934.

Dempster, A.P. (1967). 'Upper and Lower Probabilities Induced by a Multivalued Map- 
ping,' Annals of Mathematical Statistics 38, 325-339.

Dominiak, A. (2008). 'Epistemic Foundation od Decision-Making under Ambiguity,' mimeo.

EllsBerG, D. (1961). 'Risk, Ambiguity and the Savage Axioms,' Quarterly Journal of Economics 75, 643-669.

Ghirardato, P. (2002). 'Revisiting Savage in a Conditional World,' Economic Theory 20, 83-92.

Ghirardato, P. And M. Marinacci (2002). 'Ambiguity Made Precise: A Comparative Foundation,' Journal of Economic Theory 102, 251-289.

GilboA, I. AND D. Schmeidler (1994). 'Additive representations of non-additive measures and the choquet integral,' Annals of Operations Research 52, 43-65.

GilboA, I. And D. Schmeidler (1989). 'Maxmin Expected Utility with non-Unique Prior,' Journal of Mathematical Economics 18, 141-153.

Gul, F. (1992). 'Savage's Theorem with a Finite Number of States,' Journal of Economic Theory 57, 99-111.

Hammond, P.J.(1988). 'Consequentialism and the Independence Axiom,' in B.R. MuNIER (ED.) Risk, Decision and Rationality, Dordrecht: D. Reidel, 503-516.

Knight, F.H. (1921). Risk, Uncertainty, and Profit.Houghton Mifflin: New York.

Kreps, D. (1992). 'Static Choice in the Presence of Unforeseen Contingencies,' in DASGupta, P., Gale, D., Hart, O. and E. Maskin (Eds.) Economic Analysis of Markets and Games, Cambridge: MIT Press, 258-281.

Lipman, B.L. (1999). 'Decision theory without logical omniscience : toward an axiomatic framework for bounded rationality,' The Review of Economic Studies 66, 339-361.

Machina, M. (1989). 'Dynamic Consistency and Non-Expected Utility Models of Choice under Uncertainty,' Journal of Economic Literature 27, 1622-1688.

Morris, S. (1996). 'The Logic of Belief and Belief Change: A Decision Theoretic Approach,' Journal Of Economic Theory 69, 1-23.

MuKERJI, S. (1997). 'Understanding the nonadditive probability decision model,' Economic Theory $\mathbf{9}, 23-46$.

Savage, L. J. (1954). The foundations of statistics. New York: Wiley.

SchmeIDler, D. (1989). 'Subjective Probability and Expected Utility without Additivity,' Econometrica 57, 571-587.

Shafer, G. (1976). A Mathematical Theory of Evidence. Princeton: Princeton University Press. 